\title{
Photon strength function and neutron/proton capture
}

\author{
R. Reifarth, ${ }^{a, b}$ M. Heil, ${ }^{b}$ F. Käppeler ${ }^{c}$ R. Plag, ${ }^{b}$ F. Bečváŕr, ${ }^{d}$ M. Krtičkaa ${ }^{d}$ \\ E-mail: r.reifarthegsi.de \\ a GSI Darmstadt, Planckstr. 1, D-64291 Darmstadt, Germany \\ ${ }^{b}$ University, Germany \\ ${ }^{c}$ Forschungszentrum Karlsruhe, P.O. Box 3640, D-76021 Karlsruhe, Germany \\ ${ }^{d}$ Faculty of Mathematics and Physics, Charles University, CZ-180 00 Prague,Czech Republic
}

The detection of $\gamma$-rays following neutron capture is a powerful tool to gain knowledge about the photon strength function of the compound nucleus. Depending on the experimental setup, the results will be averaged over a range of neutron energies or only one resonance. For certain radioactive isotopes, the measurement of $\gamma$-rays following a proton capture in inverse kinematics might be a feasible alternative to investigate the respective compound nucleus.

Workshop on Photon Strength Functions and Related Topics

June 17-20 2007

Prague, Czech Republic 

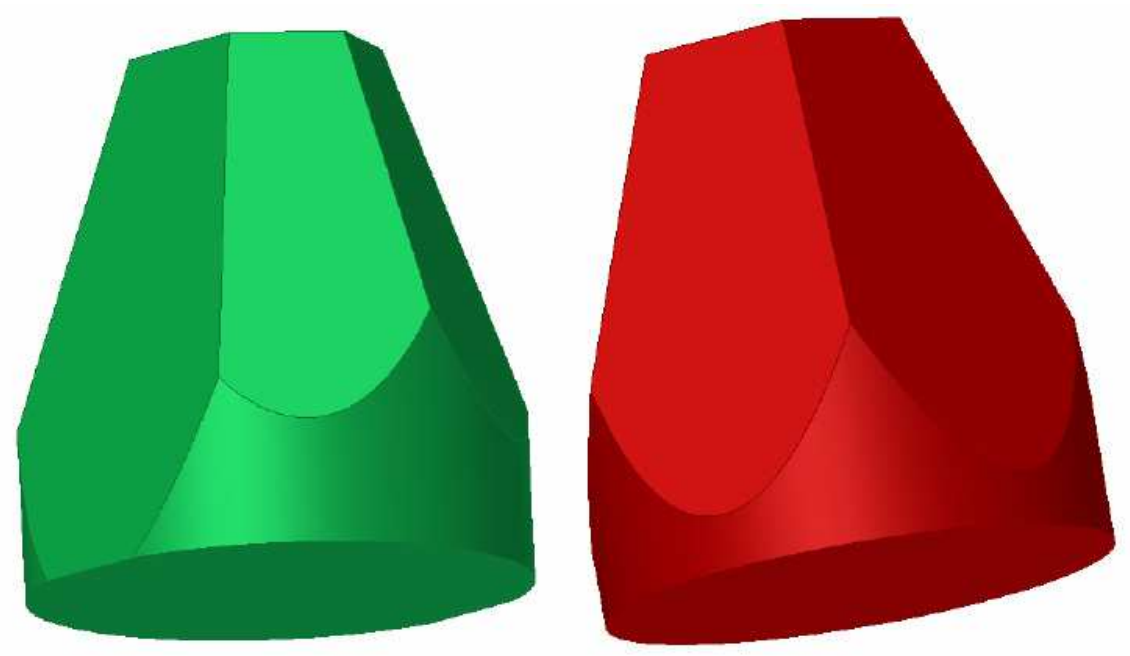

Figure 1: Cylindrically cut crystals (hexagon left; pentagon right) used for the Karlsruhe crystal ball. The complete sphere would consist of 30 hexagons and 12 pentagons.

\section{Neutron capture}

\subsection{The Karlsruhe crystal ball}

The Karlsruhe $4 \pi \mathrm{BaF}_{2}$ array is used for the determination of $(\mathrm{n}, \gamma)$ cross-sections in the astrophysically relevant neutron energy range from 3 to $220 \mathrm{keV}$. A detailed description is given by Wisshak et al. [1]. The main idea of the geometry used for the crystals is described by Habs et al. [2]. For the $\mathrm{BaF}_{2}$ crystal ball in Karlsruhe a solution with two different crystal shapes, 12 regular pentagons and 30 irregular hexagons, was realized (Figure 1). During $(\mathrm{n}, \gamma)$ experiments, one crystal is left out at the beam entrance and the opposite crystal (beam exit) has a hole. The crystal sphere has an inner radius of $10 \mathrm{~cm}$ and an outer radius of $25 \mathrm{~cm}$. In order to fit the photo multiplier tubes, the crystals are cylindrically cut with a diameter of $14 \mathrm{~cm}$ at outer the ends (Figure 1).

Compared to similar detection systems installed at neutron beams, one of the main advantages of the experimental setup of the Karlsruhe crystal ball is the possibility of discriminating neutron capture events in the sample from events resulting from neutrons scattered off the sample using time of flight information. Because of the short neutron flight path of only $80 \mathrm{~cm}$ and the tailored neutron spectrum, scattered neutrons have to travel significantly longer before they can eventually interact with surrounding material and produce $\gamma$-rays. Figure 2 shows this effect for the examples of neutron capture on ${ }^{129} \mathrm{Xe}$. Immediately after the onset of neutron capture events, there is basically no background from neutron scattering. This allows the extraction of very pure neutron capture data, which can then be compared with simulations.

The $\gamma$-ray detection efficiency of the crystal ball is less than $100 \%$. The total efficiency for mono-energetic $\gamma$-rays in the $\mathrm{MeV}$ range is about $90 \%$, and the peak efficiency is about $80 \%$. Assuming an averaged cascade multiplicity of 4 this implies on the one hand a probability for detecting the whole cascade energy of less than $40 \%$, and on the other hand a probability of less then $1 \%$ for not detecting any gamma-ray at all. This means, the total detection efficiency is 


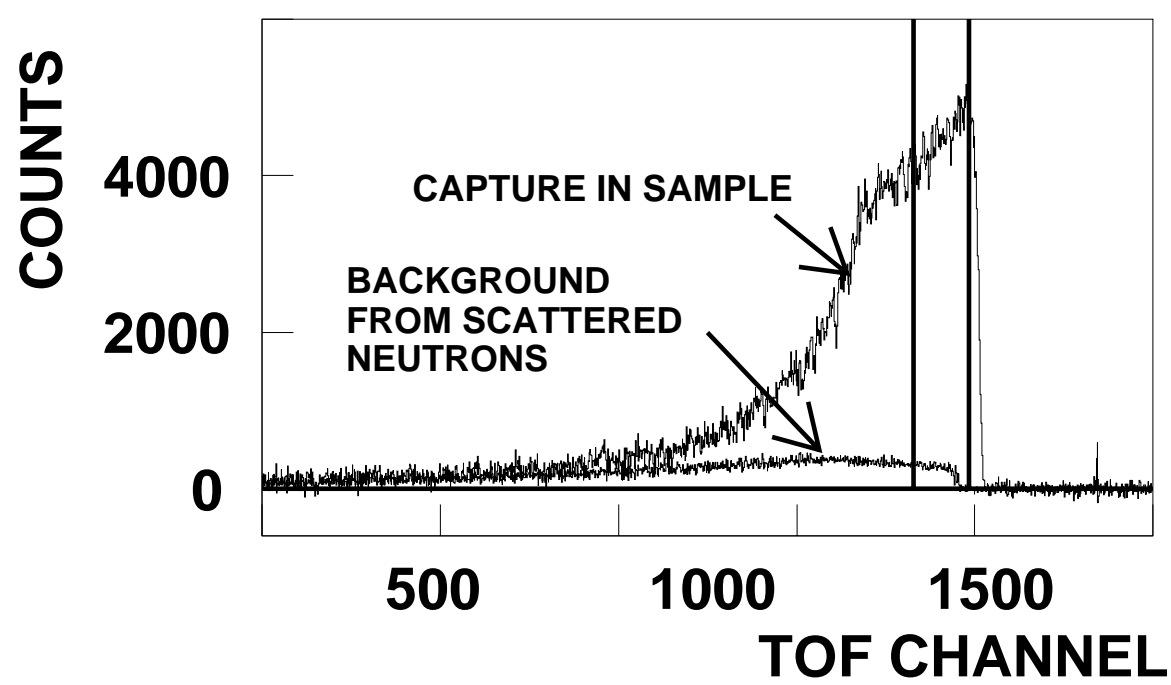

Figure 2: Time-Of-Flight (TOF) spectra measured with a ${ }^{129} \mathrm{Xe}$ samples (100 keV maximum neutron energy). The TOF increases from right to left. The sharp peak at the right end of the spectrum originates from the first interaction of the protons with the ${ }^{7} \mathrm{Li}$ target, which produces neutrons via ${ }^{7} \mathrm{Li}(\mathrm{p}, \mathrm{n})$. The background from neutrons scattered off the sample is shown separately. The region used for cross-section analysis is indicated by vertical lines [3].

$100 \%$, while the peak efficiency is significantly lower. On the other hand, applying an experimental energy threshold of $1.6 \mathrm{MeV}$ is necessary, because of the high intrinsic background of the $\mathrm{BaF}_{2}$ crystals due to the Ra-decay chain, refusing also true capture events in the sample. An accurate description of the lost part in the $\gamma$-ray spectrum is necessary to achieve uncertainties of less than $2 \%$. One motivation of the present work was to provide a completely independent way of estimating this part of missed events. For this purpose two independent simulation codes were used: the CASINO code [4], an extension of the Monte Carlo code DICEBOX [5] for generating artificial $\gamma$-cascades following the capture of $\mathrm{keV}$ mono-energetic proved neutrons, and the implementation of the GEANT code [6] for imitating the response of the $4 \pi \mathrm{BaF}_{2}$ detector to these cascades.

The detector simulation tool GEANT 3.21 [6] was developed at CERN in order to simulate complex detector systems used in high energy physics. With some restrictions it is possible to use this program to simulate neutron capture experiments, were energies of only a few $\mathrm{MeV}$ per event are released. A first test simulating the response to mono-energetic $\gamma$-rays proved the feasibility of the tool for this purpose (Figure 3).

In our approach the CASINO simulations yield sets of transition energies for individual steps of a large number of cascades. While simulating these cascades the role of electron conversion is properly taken into account, which makes it possible to mark the transitions in each set by a flag distinguishing between $\gamma$-rays and electrons. All this outcome of the CASINO simulations is in turn used as an input for the subsequent GEANT simulations to predict the observables of interest, specifically the instrumental sum energy $\gamma$-ray spectra for individual detector multiplicities. With the requirement that these observables are reproducing the experimental data, the needed correction for not observed capture events can be determined. 


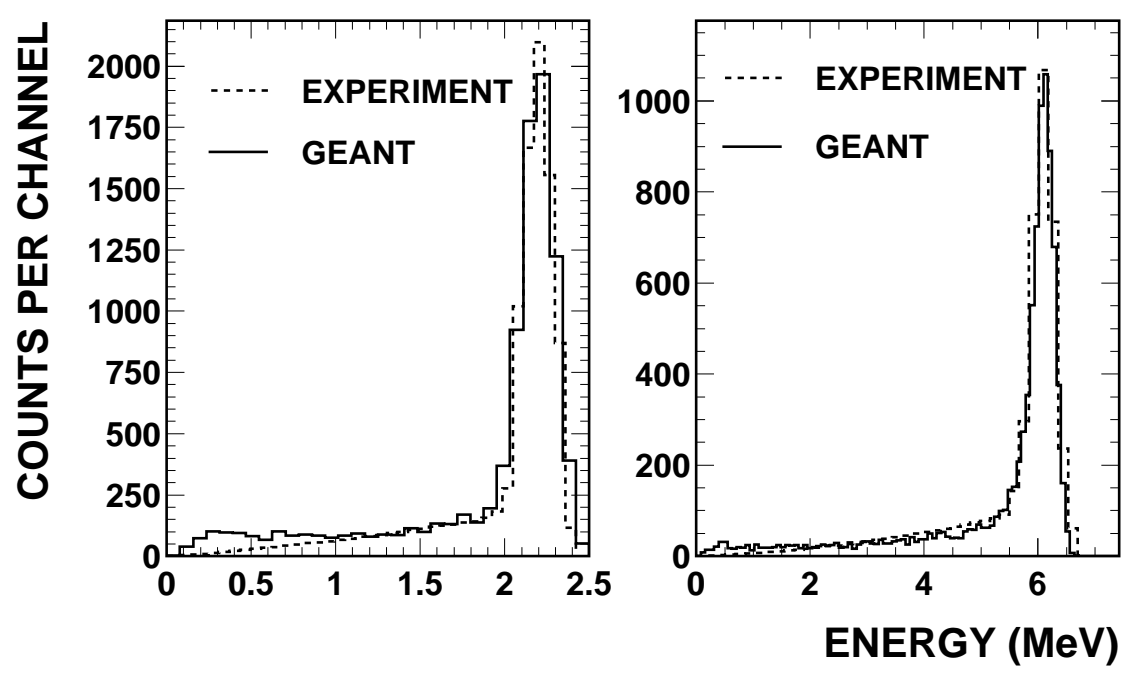

Figure 3: Comparison of simulated (solid line) and measured (dashed line) sum energy spectra of the Karlsruhe $4 \pi$-calorimeter to mono-energetic $\gamma$-rays with $2.209 \mathrm{MeV}$ (left) and 6.146 MeV (right).

The DICEBOX algorithm embodies the basic postulates of the extreme statistical model of nuclei and the paradigm of the photon strength functions [7]. The CASINO code [4] mimics the Markovian process of the emission of the $\gamma$-cascades and allows us to keep a full control over the influence of the Porter-Thomas fluctuations of the reduced neutron widths of the resonance capturing states together with fluctuations of all partial radiation widths involved. The energy profile of the incident $\mathrm{keV}$ neutron beam is taken into account, as well as the neutron penetrabilities and the strength functions for $s$ - and $p$-wave neutrons. Because of scarcity of the data on photon strength functions in the mass region near $A=130$, we carried out separate CASINO simulations assuming several combinations of existing models for these quantities. For the $E 1$ strength we used the Brink-Axel (BA) model [8], the Fermi-liquid model according to Kadmenskij, Markushev and Furman (KMF) [9] or a mutation of this model, known as GLO model, see Refs. [10]. These models were combined with the well-known $M 1$ single-particle (SP) model with a constant photon strength or an alternative model that assumes spin-flip $M 1$ vibrations, see e.g. [11]. For simulation of level energies in the quasi-continuum we used either the constant-temperature leveldensity formula (CTF) or the formula following from the back-shifted Fermi gas (BSFG) model, see Ref. [12]. Below some excitation energy we fully utilized the existing experimental data on level energies and intensity branching ratios [13].

Figure 4 shows a comparison of two different sets of ${ }^{197} \mathrm{Au}(\mathrm{n}, \gamma)$ cascades with experimental data. It underlines the power of a segmented calorimeter, since models resulting in good fits can clearly be distinguished from models resulting in bad fits. The difference in the two models used for this comparison were different parameters for the photon strength function of the ${ }^{198} \mathrm{Au} \mathrm{nu}-$ cleus. Figure 5 shows a similar comparison, but only for the good model shown in Figure 4 . The difference between the two simulated curves is that the effect of the electron conversion was turned on or off. It is obvious that the electron conversion has to be taken into account in order to describe 

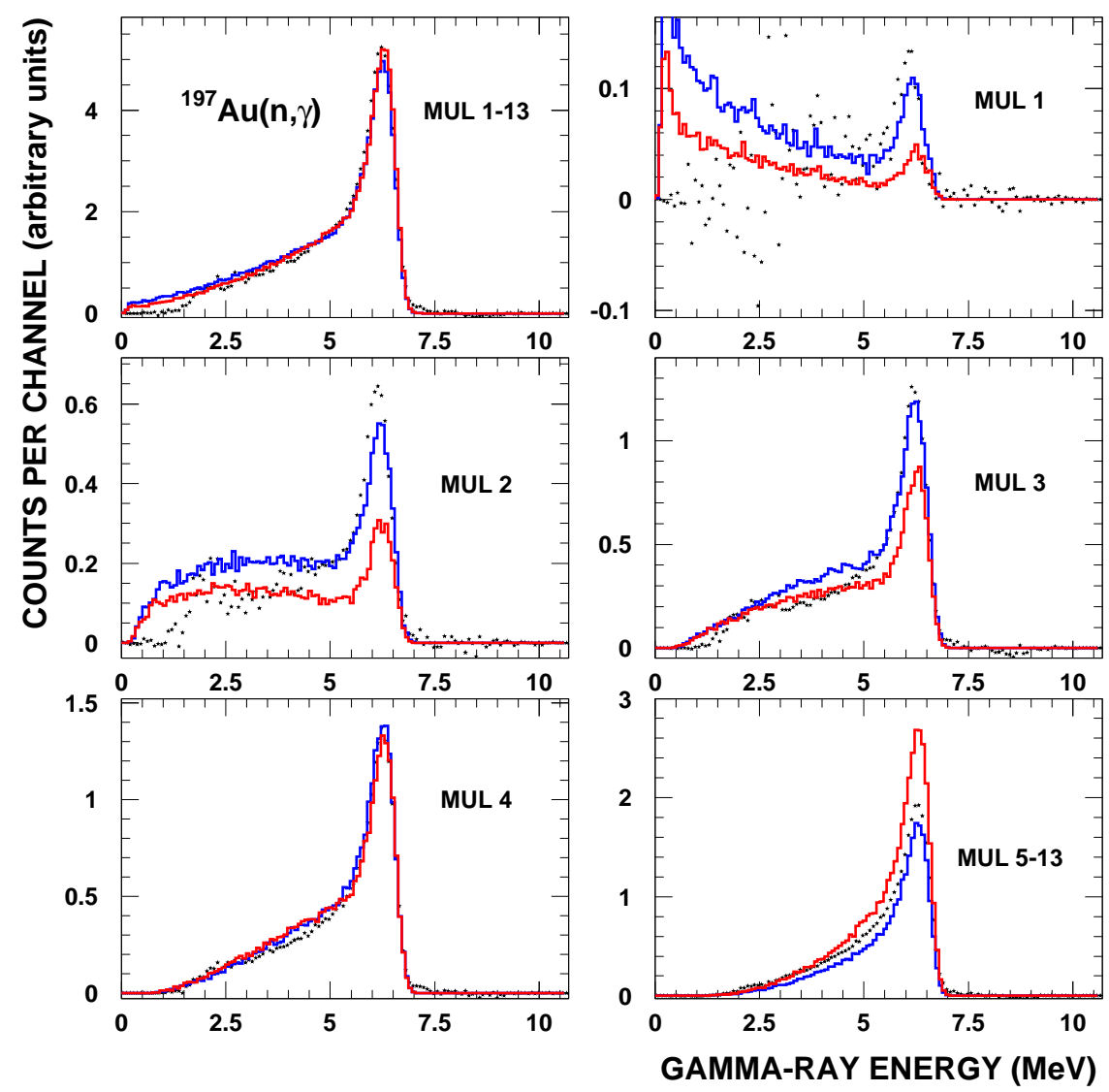

Figure 4: Comparison of experimental sum energy spectra with simulated cascades for ${ }^{197} \mathrm{Au}(\mathrm{n}, \gamma)$ from [4] for different multiplicities. The abbreviation MUL stands for multiplicity. Parameter set resulting in good fit (blue) and poor fit (red).

the experimental data. The "electron-converted" $\gamma$-rays are lost for practically all $\gamma$-ray detectors, since the electrons will deposit their energy in the sample or detector wrapping and not in the active material. Such transitions basically reduce the experimentally detectable Q-value.

Figure 6 shows a comparison of two different sets of ${ }^{155} \mathrm{Gd}(\mathrm{n}, \gamma)$ cascades with experimental data. It highlights again the importance of a segmentation, since both sets describe the total sum energy spectrum fairly well. But they disagree significantly, if sum energy spectra with multiplicity cuts are compared.

\subsection{The DANCE array}

The Detector for Advanced Neutron Capture Experiments (DANCE) is designed as a high efficiency, highly segmented $4 \pi \mathrm{BaF}_{2}$ detector for calorimetrically detecting $\gamma$-rays following a neutron capture. DANCE is located on the 20 m neutron flight path 14 (FP14) at the Manuel Lujan Jr. Neutron Scattering Center at the Los Alamos Neutron Science Center (LANSCE) [14]. The initial design work is described in [15]. For practical reasons the detector modules do not really 

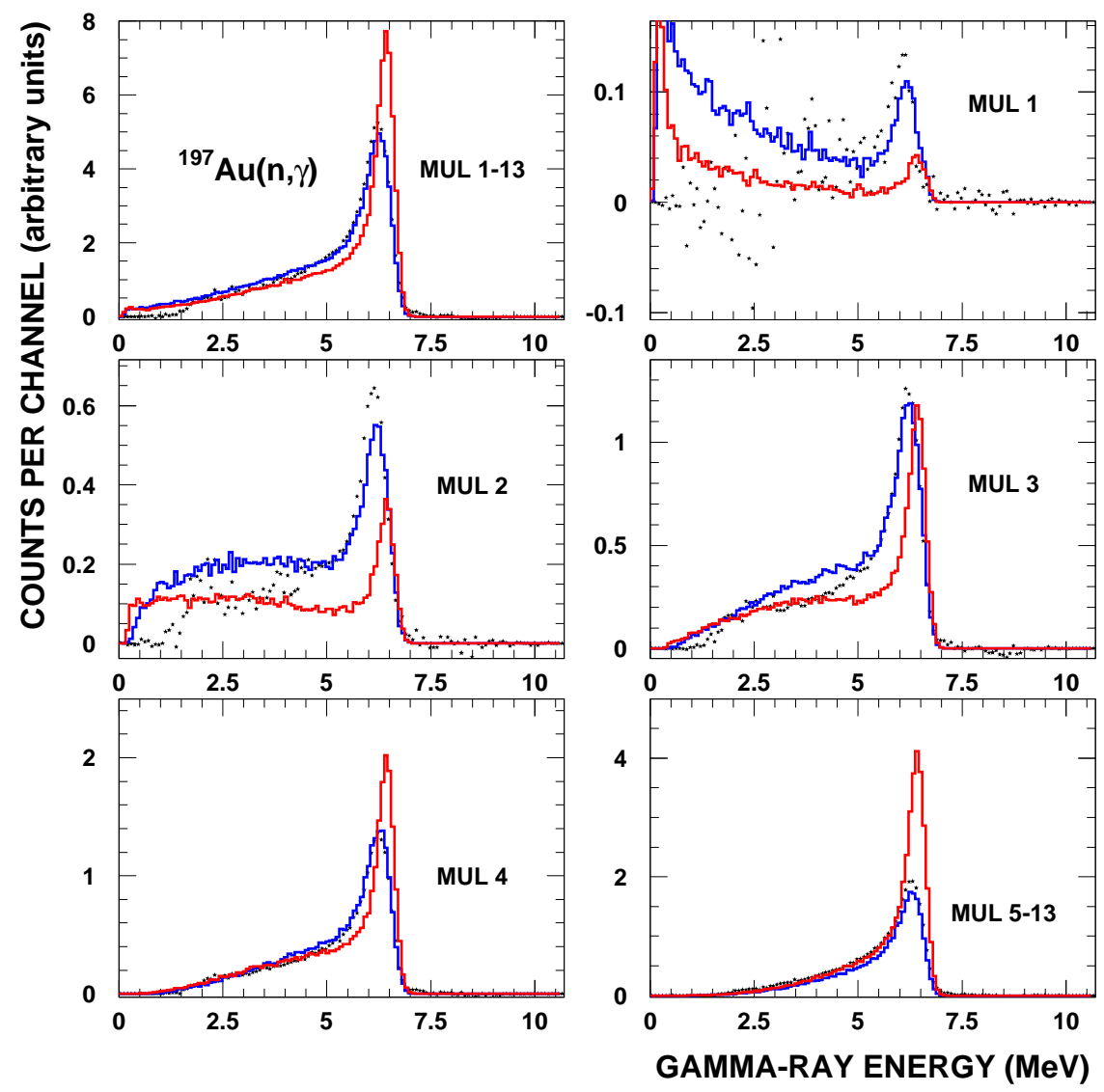

Figure 5: Comparison of experimental sum energy spectra with simulated cascades for ${ }^{197} \mathrm{Au}(\mathrm{n}, \gamma)$ from [4] for different multiplicities with (blue) and without (red) electron conversion. The abbreviation MUL stands for multiplicity.

cover the entire solid angle. The design of the detector is such that a full $4 \pi$ array would consist of 162 crystals of four different shapes, each shape covering the same solid angle [2]. Two of the 162 crystals are left out in order to leave space for the neutron beam pipe. Depending on the experiment, one crystal can be replaced by a sample changer mechanism, which makes it possible to exchange up to 3 samples without closing the beam shutter and breaking the vacuum of the beam pipe. Thus the full array is designed to host 159 or 160 out of 162 possible $\mathrm{BaF}_{2}$ crystals. The dimensions of the bare crystals are designed to form a $\mathrm{BaF}_{2}$ shell with an inner radius of $17 \mathrm{~cm}$ and a thickness of $15 \mathrm{~cm}$. Thanks to the fairly low repetition rate of $20 \mathrm{~Hz}$, measurements can be carried out over the whole energy range from $10 \mathrm{meV}$ to $500 \mathrm{keV}$. This combination of a strong neutron source and a high efficiency $\gamma$-ray detector allows to measure $(\mathrm{n}, \gamma)$ cross sections of radioactive isotopes down to a few hundred days half-life. Further details on the overall performance of the array can be found in [16, 17].

Since the neutron source at LANSCE is a white source, meaning that neutron energies over a wide range are available, neutron capture events can, in principle, also be investigated over a wide 


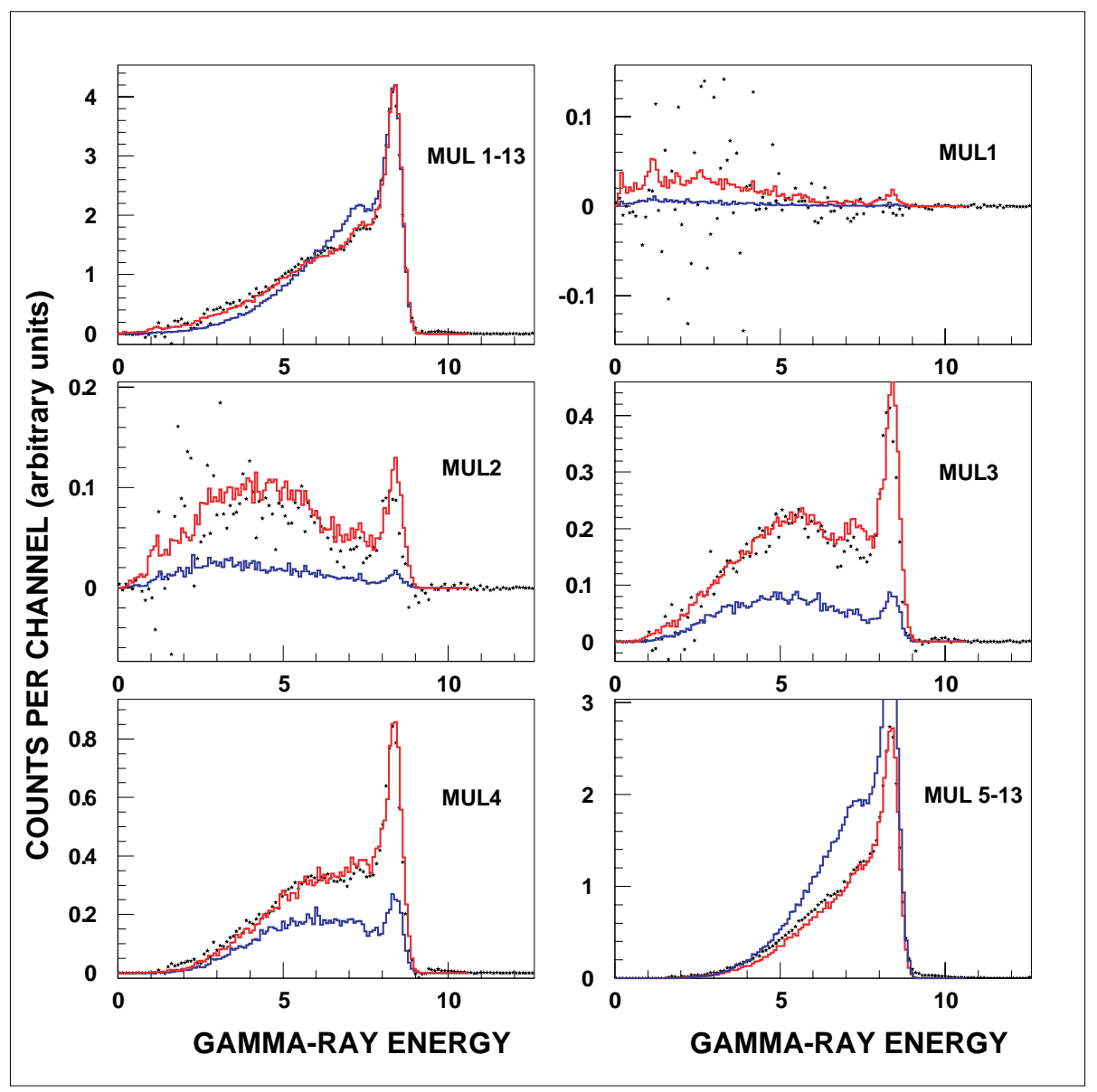

Figure 6: Comparison of experimental sum energy spectra with simulated cascades for ${ }^{155} \mathrm{Gd}(\mathrm{n}, \gamma)$ from [4] for different multiplicities. The abbreviation MUL stands for multiplicity. Parameter set resulting in good fit (red) and poor fit (blue), even though both describe the sum over all multiplicities correctly.

range. On the other hand however, the discrimination against neutron capture in other materials than the sample under investigation can be much more difficult.

Figure 7 shows the yield of a measurement with a ${ }^{151} \mathrm{Sm}$ sample $\left(t_{1 / 2}=93\right.$ years $)$ measured with the DANCE array in the thermal neutron energy region. Clearly, there are regions around the resonances and under the thermal peak, where a basically background-free measurement is possible. In the valleys between the resonances however, the background from scattered neutrons can even be dominating the total yield.

This effect is even more pronounced, if higher neutron energies are under investigation because the ratio of capture to scatter cross section becomes smaller. Figure 8 shows a similar plot like Figure 7, but for ${ }^{237} \mathrm{~Np}(\mathrm{n}, \gamma)\left(t_{1 / 2}=2.1 \cdot 10^{6}\right.$ years $)$ and for higher energies. Here an analysis of the capture cascades is only possible at the resonances. The advantage of the long flight path is however, that the neutron-energy resolution is sufficient to analyze neutron capture data for each resonance individually. While varying the neutron energy from resonance to resonance does not change the energy of the initial state of the compound nucleus significantly, it may change the 


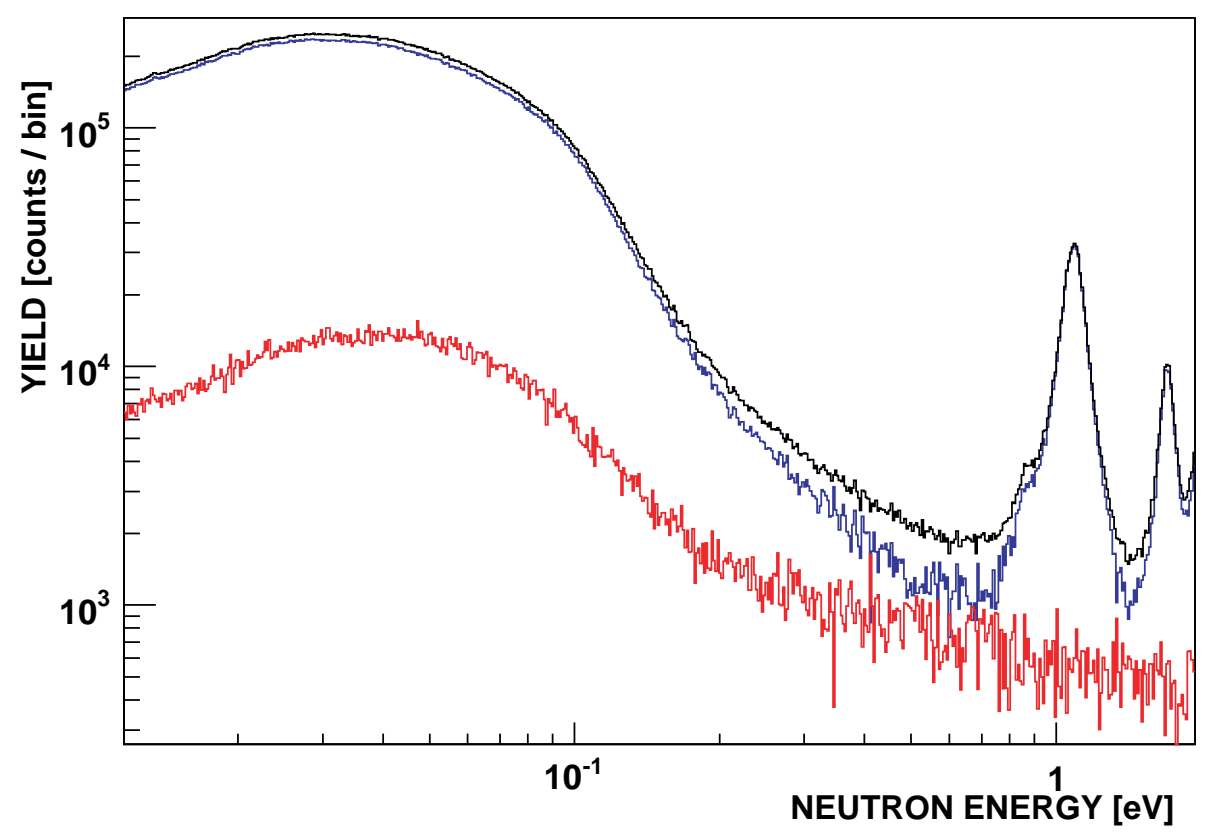

Figure 7: Measured counts (black), ${ }^{151} \mathrm{Sm}$ yield (blue), background (red) for thermal neutron energies.

initial spin, which may result in significantly different cascades [18].

\section{Proton capture}

Radioactive beams offer the opportunity to extend the experimentally based knowledge about nuclear structure far beyond the valley of stability. Especially within the planned international Facility for Antiproton and Ion Research (FAIR) at GSI [19], radioactive ions will be produced with highest intensities. It is very often not feasible to collect the respective radioactive ions in order to produce a sample for irradiation with e.g. neutrons as described in the preceding chapter. Experiments in inverse kinematics - irradiating a stable target with the desired radioactive ions - are the solution to that problem. Neutron targets however, are so far also not feasible with the required neutron densities. It is therefore necessary to consider different reactions, like $(\mathrm{p}, \gamma)$, to investigate the unstable nuclei. Two collaborations are already established to perform a variety of experiments at the external target $\left(\mathrm{R}^{3} \mathrm{~B}\right)$ and the new experimental storage ring (EXL) of the new FAIR facility.

The proposed $\mathrm{R}^{3} \mathrm{~B}$, a universal setup for kinematically complete measurements of Reactions with Relativistic Radioactive Beams (Figure 9) will cover experimental reaction studies with exotic nuclei far off stability, with emphasis on nuclear structure and dynamics. Astrophysical aspects and technical applications are also concerned. $\mathrm{R}^{3} \mathrm{~B}$ is a versatile reaction setup with high efficiency, acceptance, and resolution for kinematically complete measurements of reactions with high-energy radioactive beams. The setup will be located at the focal plane of the high-energy branch of the Super-FRS. The experimental configuration is based on a concept similar to the existing LAND reaction setup at GSI introducing substantial improvement with respect to resolution and an extended detection scheme, which comprises the additional detection of light (target-like) recoil particles and 


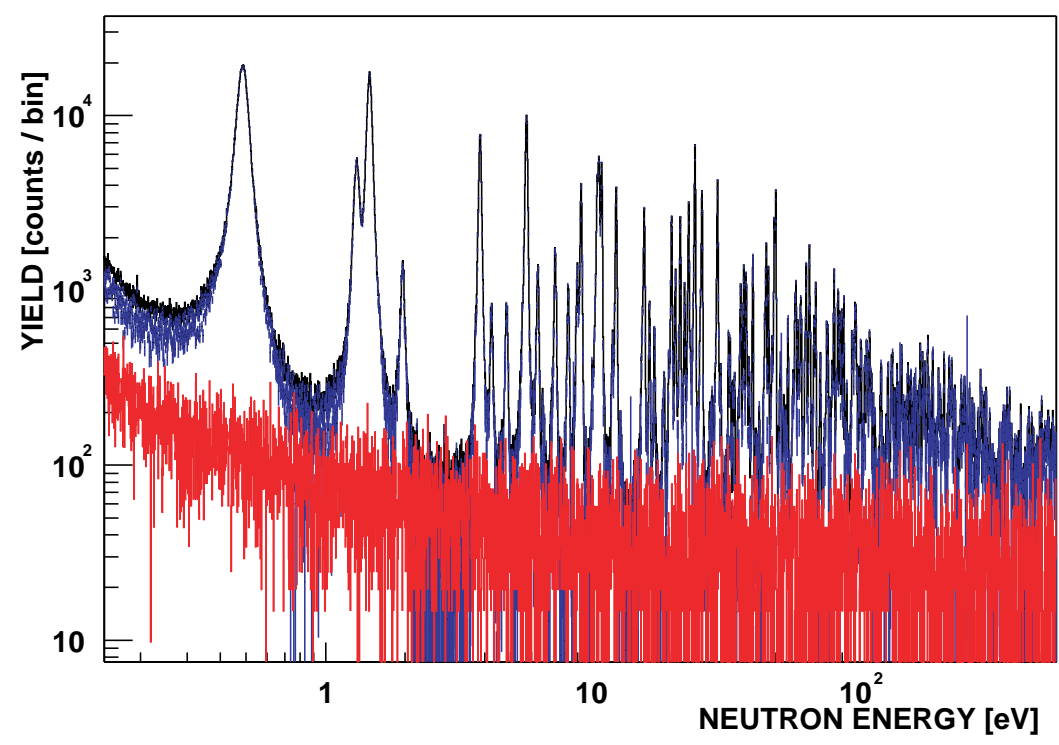

Figure 8: Measured counts (black), ${ }^{237} \mathrm{~Np}$ yield (blue), background (red) for low neutron energies.

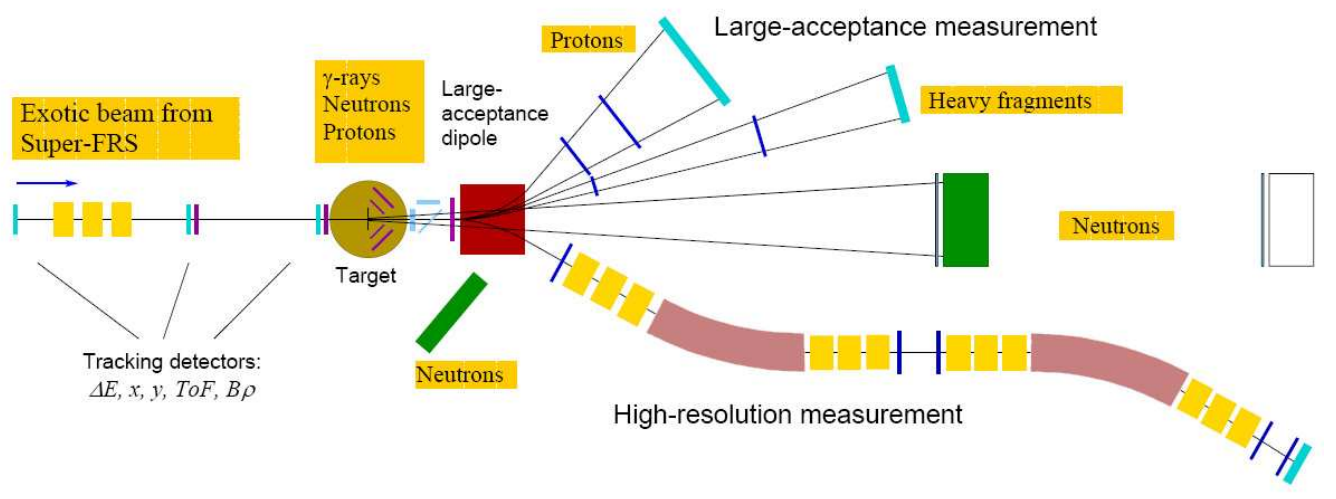

Figure 9: Schematic drawing of the $\mathrm{R}^{3} \mathrm{~B}$ setup comprising $\gamma$-ray and target recoil detection, a largeacceptance dipole magnet, a high-resolution magnetic spectrometer, neutron and light-charged particle detectors, and a variety of heavy-ion detectors [20].

a high-resolution fragment spectrometer. The setup is adapted to the highest beam energies (corresponding to $20 \mathrm{Tm}$ magnetic rigidity) provided by the Super-FRS capitalizing on the highest possible transmission of secondary beams. The experimental setup is suitable for a wide variety of scattering experiments, i.e., such as heavy-ion induced electromagnetic excitation, knockout and breakup reactions, or light-ion (in)elastic and quasi-free scattering in inverse kinematics, thus enabling a broad physics program with rare-isotope beams to be performed [20]. In order to investigate $(\mathrm{p}, \gamma)$ reactions in inverse kinematics, the target could be polyethylene $\left(\mathrm{CH}_{2}\right)$ or liquid hydrogen $\left(\mathrm{LH}_{2}\right)$. The energy of the radioactive ions can range between 100 and $1000 \mathrm{MeV} / \mathrm{A}$. 

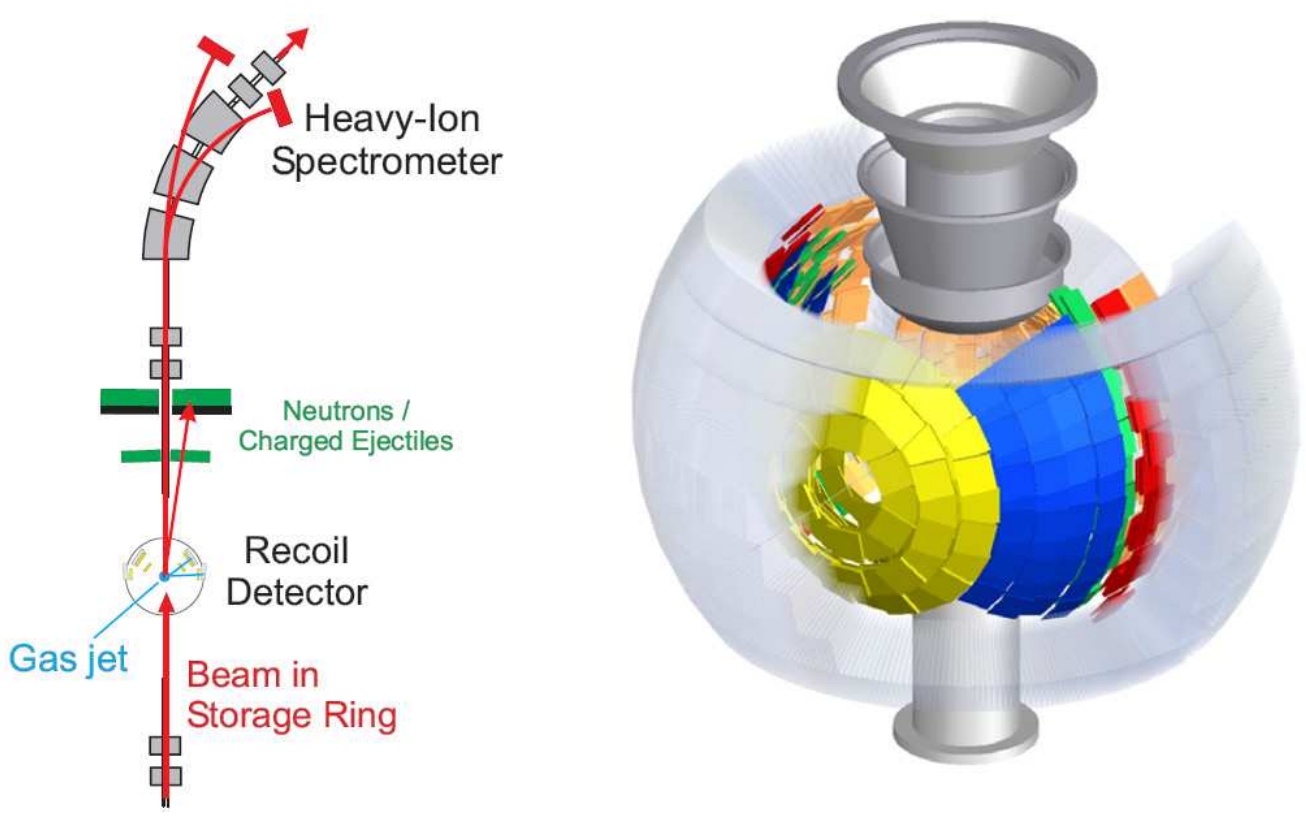

Figure 10: Schematic view of the EXL detection systems. Left: Set-up built into the NESR storage ring. Right: Target-recoil detector surrounding the gas-jet target [21].

The EXL objective is to capitalize on light-ion reactions in inverse kinematics by using novel storage-ring techniques and a universal detector system providing high resolution and large solid angle coverage in kinematically complete measurements. The apparatus shown in Figure 10 is planned to be installed at the internal target at the NESR storage-cooler ring at FAIR. It comprises a silicon target-recoil detector for charged particles, completed by $\gamma$-ray and slow-neutron detectors, located around the internal gas-jet target, forward detectors for fast ejectiles (both charged particles and neutrons), and an in-ring heavy-ion spectrometer [21]. The radioactive ions will interact with the gas target $(\mathrm{H}, \mathrm{He})$ at intermediate energies $(10-500 \mathrm{MeV} / \mathrm{A})$.

\section{Acknowledgments}

R.R. and R.P. are supported by the HGF Young Investigators Project VH-NG-327.

\section{References}

[1] K. Wisshak, K. Guber, F. Käppeler, J. Krisch, H. Müller, G. Rupp, F. Voss. The Karlsruhe $4 \pi$ Barium Fluoride Detector. Nucl. Instr. Meth. A 292 (1990) 595.

[2] D. Habs, F. S. Stephens, R. M. Diamond. A Proposal for a Crystal Ball Detector System. Tech. rep., Report LBL-8945, Lawrence Berkeley Laboratory (1979).

[3] R. Reifarth, M. Heil, F. Käppeler, F. Voss, K. Wisshak, F. Bečvár, M. Krtička, R. Gallino, Y. Nagai. The stellar neutron capture cross sections of ${ }^{128,129,130}$ Xe. Phys. Rev. C (Nuclear Physics) $6 \mathbf{6 6}(6)$ 064603. 
[4] F. Bečvář. Statistical $\gamma$ Cascades Following Thermal and keV Neutron Capture in Heavy Nuclei. In S. Wender, ed., Gamma-Ray Spectroscopy and Related Topics. American Institute of Physics, New York (2000), 504.

[5] F. Bečvář. Simulation of $\gamma$ cascades in complex nuclei with emphasis on assessment of uncertainties of cascade-related quantities. Nucl. Instr. Meth. A 417 (1998) 434.

[6] J. Apostolakis. CERN program library long writeup, W5013. Tech. rep., CERN, GEANT library (1993). Http://wwwinfo.cern.ch/asd/geant/.

[7] G. A. Bartholomew. Neutron Capture Gamma Rays. Annual Review of Nuclear Science 11 (1961) 259.

[8] P. Axel. Electric dipole ground-state transition width strength function and 7-MeV photon interactions. Physical Review 126 (1962) 671.

[9] S. G. Kadmenskii, V. P. Markushev, V. I. Furman. Radiative Widths of Neutron Resonances Giant-Dipole Resonances. Soviet Journal of Nuclear Physics-USSR 37 (1983) 165.

[10] J. Kopecky, M. Uhl, R. E. Chrien. Radiative Strength in the Compound Nucleus Gd-157. Physical Review C 47 (1993) 312.

[11] D. Frekers, H. J. Wortche, A. Richter, R. Abegg, R. E. Azuma, A. Celler, C. Chan, T. E. Drake, R. Helmer, K. P. Jackson, J. D. King, C. A. Miller, R. Schubank, M. C. Vetterli, S. Yen. Spin Excitations in the Deformed-Nuclei Sm-154, Gd-158 and Er-168. Physica Letters B 244 (1990) 178.

[12] T. von Egidy, T., H. Schmidt, A. Behkami. Nuclear level densities and level spacing distributions: Part II,. Nucl. Phys. A 481 (1988) 189.

[13] ENDF-LIBRARY. http://www.nndc.bnl.gov/nndc/endf/. Tech. rep., National Nuclear Data Center, On-Line Access: www.nndc.bnl.gov/nndc/endf/; Brookhaven National Laboratory (2001).

[14] P. W. Lisowski, C. D. Bowman, G. J. Russell, S. A. Wender. The Los Alamos National Laboratory Spallation Neutron Sources. Nucl. Sci. Engineering 106 (1990) 208.

[15] M. Heil, R. Reifarth, M. M. Fowler, R. C. Haight, F. Käppeler, R. S. Rundberg, E. H. Seabury, J. L. Ullmann, J. B. Wilhelmy, K. Wisshak, F. Voss. A $4 \pi B a F_{2}$ detector for $(n, \gamma)$ cross-section measurements at a spallation neutron source. Nucl. Instr. Meth. A 459 (2001) 229.

[16] R. Reifarth, T. A. Bredeweg, A. Alpizar-Vicente, J. C. Browne, E.-I. Esch, U. Greife, R. C. Haight, R. Hatarik, A. Kronenberg, J. M. O’Donnell, R. S. Rundberg, J. L. Ullmann, D. J. Vieira, J. B. Wilhelmy, J. M. Wouters. Background identification and suppression for the measurement of $(n, \gamma)$ reactions with the DANCE array at LANSCE. Nucl. Instr. Meth. A 531 (2004) 528.

[17] J. L. Ullmann, U. Agvaanluvsan, A. Alpizar-Vicente, E. Bond, T. A. Bredeweg, et al. DANCE overview. AIP conference proceedings 769 (2005) 918.

[18] P. E. Koehler, J. L. Ullmann, T. A. Bredeweg, J. M. O’Donnell, R. Reifarth, R. S. Rundberg, D. J. Vieira, J. M. Wouters. Spin measurements for Sm147+n resonances: Further evidence for nonstatistical effects. Physical Review C 76 (2007) 025804.

[19] W. F. Henning. An International Accelerator Facility for Beams of Ions and Antiprotons - Conceptual Design Report. Tech. rep., GSI (2001). Http://www.gsi.de/GSI-Future/cdr/.

[20] $\mathrm{R}^{3} \mathrm{~B}$-collaboration. Technical Proposal for the Design, Construction, Commissioning and Operation of $R^{3} B$. Tech. rep., GSI (2005). Http://www-land.gsi.de/r3b/. 
[21] EXL-Collaboration. Technical Proposal for the Design, Construction, Commissioning and Operation of the EXL Project (Exotic nuclei studied in light-ion induced reactions at the NESR storage ring). Tech. rep., GSI (2005). Http://ns.ph.liv.ac.uk/ mc/EXL/ns-instrum-exl.html/. 